\title{
Mitochondria-targeted inhibitors of the human SIRT3 lysine deacetylase $\dagger$
}

Kathrin S. Troelsen, Michael Bæk, Alexander L. Nielsen, Andreas S. Madsen, $\ddagger$ Nima Rajabi,§ and Christian A. Olsen*

Center for Biopharmaceuticals \& Department of Drug Design and Pharmacology, Faculty of Health and Medical Sciences, University of Copenhagen, Universitetsparken 2, DK-2100, Copenhagen, Denmark.

*cao@sund.ku.dk

† Electronic supplementary information (ESI) available.

‡ Present address A.S.M. is now at Novo Nordisk A/S, DK-2880, Bagsværd, Denmark.

$\S$ Present address N.R. is now at Red Glead Discovery, Lund, Sweden. 


\section{ABSTRACT}

Sirtuin 3 (SIRT3) is the major protein lysine deacetylase in the mitochondria. This hydrolase regulates a wide range of metabolically involved enzymes and has been considered as a potential drug target in certain cancers. Investigation of pharmacological intervention has been challenging due to a lack of potent and selective inhibitors of SIRT3. Here, we developed a strategy for selective inhibition of SIRT3 in cells, over its structurally similar isozymes that localize primarily to the nucleus (SIRT1) and the cytosol (SIRT2). This was achieved by directing the inhibitors to the mitochondria through incorporation of mitochondria-targeting peptide sequences into the inhibitor structures. Our inhibitors exhibited excellent mitochondrial localization in HeLa cells as indicated by fluorophoreconjugated versions, and target engagement was demonstrated by a cellular thermal shift assay of SIRT3 using western blotting. The acetylation state of documented SIRT3 target MnSOD was shown to be increased in cells with little effect on known targets of SIRT1 and SIRT2, showing that our lead compound exhibits selectivity for SIRT3 in cells. We expect that the developed inhibitor will now enable a more detailed investigation of SIRT3 as a potential drug target and help shed further light on the diverse biology regulated by this enzyme.

Keywords: Sirtuins, SIRT3, histone deacetylase inhibitors, mitochondrial targeting, cell penetrating peptides 


\section{Introduction}

The sirtuin (SIRT) enzymes are evolutionarily conserved hydrolases of the class III lysine deacetylases (KDACs), cleaving acyl-based posttranslational modifications (PTMs) on lysine side chains in the proteome. The human genome encodes seven sirtuin isoforms, SIRT1-7, which have different substrate specificities, cellular localization, and tissue-dependent expression levels. ${ }^{1}$ The major deacetylases [i.e., targeting the $\varepsilon-N$-acetyllysine (Kac) PTM] are SIRT1-3, SIRT6, and SIRT7, of which the class I sirtuins, SIRT1-3, share the highest similarity in substrate specificity. ${ }^{2,3}$ Thus, SIRT1-3 and 6 efficiently hydrolyze Kac residues as well as longer hydrocarbon-based $\varepsilon-N$ acyllysine PTMs such as $\varepsilon-N$-myristoyllysine $(\mathrm{Kmyr})^{4-8}$ to regulate diverse biological function, including metabolic homeostasis and health span. ${ }^{9}$ However, the class I sirtuins have also been implicated in the pathogenesis of various diseases and, depending on the condition, either activation or inhibition of SIRT1-3 have been considered as potential therapeutic strategies to treat several cancers and neurodegenerative disorders. ${ }^{10-12}$ The SIRT3 isoform is the only mitochondrially localized sirtuin that exhibits potent deacetylase activity, with other mitochondrial sirtuins, SIRT4 and SIRT5, mainly targeting negatively charged PTMs. ${ }^{13-19}$ Here, SIRT3 regulates a number of metabolic enzymes involved in the respiratory chain, ${ }^{20}$ TCA cycle, ${ }^{21,} 22$ fatty acid $\beta$-oxidation, ${ }^{23}$ and ketogenesis. $^{24}$ Furthermore, it controls mitochondrial oxidative pathways by regulating the production of reactive oxygen species (ROS), ${ }^{25}$ e.g. through activation of manganese superoxide dismutase (MnSOD). ${ }^{26,27}$

The sirtuins share a common deacylase mechanism, which is dependent on the co-substrate nicotinamide adenine dinucleotide (NAD+). This mechanism has been utilized to develop so-called mechanism-based inhibitors, by the use of substrate-mimicking chemotypes that form stalled intermediates in the active site of the sirtuin. ${ }^{28-35}$ Many mechanism-based inhibitors exhibit high potency and, in several cases, high selectivity toward specific sirtuin subtypes. However, due to the shared mechanism and similar substrate preferences between SIRT1-3, it has been difficult to target SIRT3 selectively. Therefore, we envisioned adopting a strategy to achieve selective targeting of the enzyme through specific subcellular localization of the inhibitor, rather than solely relying on selective enzyme recognition. Among several demonstrated examples of mitochondrial targeting of various payloads, ${ }^{36}$ a particularly appealing approach for our strategy was the mitochondria-targeting peptides developed by Kelley and co-workers. ${ }^{37-39}$ Based on recent investigations of mechanismbased peptide inhibitors of other sirtuins, ${ }^{32,35}$ we hypothesized that mitochondria-targeting peptide tags could be elaborated into potent inhibitors of the SIRT3 that would exhibit selectivity in cells (Figure 1). By designing such chemotypes and optimizing their selectivity profiles to inhibit only 
SIRT1-3 and not inhibit other sirtuins or HDACs, we could demonstrate selective inhibition of SIRT3 in cultured cells as well as target engagement illustrated by cellular thermal shift assays.

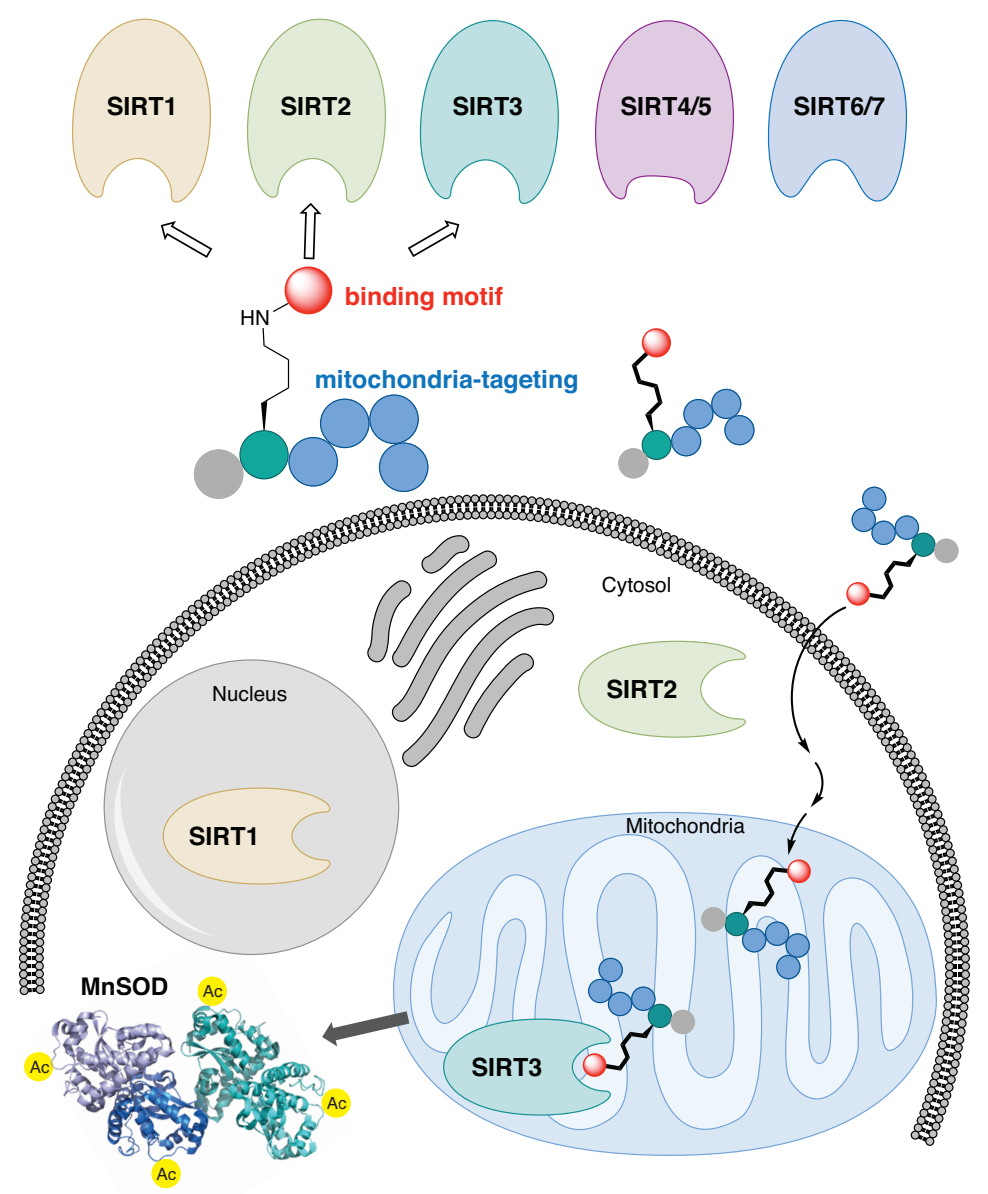

Figure 1. Schematic representation of the developed concept for inhibition of SIRT3. A potent inhibitor that exhibit class I sirtuin selectivity, inhibiting SIRT1-3, is targeted to the mitochondria to inhibit SIRT3 selectively in cells by specific localization.

\section{Results and Discussion}

\section{Structure-activity relationship study}

We envisioned that the mitochondria-targeting peptides developed by Kelley and co-workers ${ }^{37-39}$ could be starting points for mechanism-based inhibitors of sirtuins. It was hypothesized that such inhibitors could be designed by incorporating a thiocarbonyl-containing lysine residue known to enable sirtuin inhibition. 3, 29, 40, 41 Based on insight from previous structure-activity relationship (SAR) studies targeting SIRT2 and SIRT5, including selectivity profiling and co-crystal structures, ${ }^{32,}{ }^{35}$ we positioned the thiocarbonyl-containing lysine residue as the $\mathrm{N}$-terminal amino acid. With a preliminary series of compounds, we addressed the length of the mitochondria-targeting peptide 
combined with $\varepsilon-N$-thioacetyled or $\varepsilon-N$-thiomyristoylated $N$-terminal lysine residues (see Scheme $S 1$ and S2 for syntheses). These inhibitors were evaluated for their ability to inhibit the deacetylation activities of SIRT1-3, applying previously described fluorescence-based assay protocols. ${ }^{14,} 42$ Gratifyingly, this revealed that the peptide scaffolds allowed for potent inhibition of SIRT1-3 (Figure S1). Alternation of the hydrophobic and cationic residues in the mitochondria-targeting sequence, resulted in slightly increased selectivity for SIRT1 and further analogues with this architecture were not pursued (Figure S1). Based on this initial compound series, the thiomyristoylated analogs were abandoned due to their apparent selectivity towards SIRT2, which was not surprising based on previous literature. ${ }^{43}, 44 \mathrm{~A}$ small series of substitutions of the $\mathrm{N}$-terminal functional group were selected based on previous SAR studies and X-ray co-crystal structures, which indicated a high degree of freedom for the selection of functionalities at this position (Figure S2). ${ }^{32,}{ }^{35}$ Based on this series, we proceeded with the 3-phenylpropionyl group (c; Figure 2) and the alkyne-containing group (a, Figure 2, Scheme S6), which is amenable for incorporation of fluorophores or other tags using $\mathrm{Cu}(\mathrm{I})$-catalyzed azide-alkyne Huisgen $3+2$ cycloaddition "click" chemistry. ${ }^{45,} 46$ Inspired by previous studies of SIRT1-329, 35, 47-51 and the structures of the active sites in SIRT1-3 (Figure S3), we next analyzed a number of thiocarbonyl binding motifs (1-10; Figure 2A). The most potent inhibitors of SIRT3, which did not at the same time inhibit the other two isoforms to a more substantial extent, were compounds 1 and 4 (Figure 2B). Because $\varepsilon-N$-thioacetyllysine residues have been shown to be processed by SIRT1-349 and HDAC8, ${ }^{52}$ the $\varepsilon-N$ '-methylthiourea functionality (4) was chosen for further investigations.

Satisfied that potent inhibition of SIRT3 could be achieved with compound 4 without significant selectivity towards either SIRT1 or SIRT2 (Figure S4A,S5), we synthesized the compound series 11-17 (Figure 2C,D). This series combines alkyne tag- (a), phenylpropionyl- (b), and fluorescent nitrobenzoxadiazole (NBD, b)-containing $\mathrm{N}$-termini with peptide scaffolds of trimer, pentamer, and heptamer length.

The NBD fluorophore-containing series $(\mathbf{1 1}, \mathbf{1 3}, \mathbf{1 6})$, for evaluation of mitochondrial localization, was further supplemented with analogs of the heptamer containing Abz, BODIPY, ATTO, and EDANS to evaluate the effect of the fluorophore on cell entry (see Scheme S2B for structures). Before analyzing the mitochondria-targeting ability of the fluorophore-containing analogs we demonstrated excellent stability of selected inhibitors in DMEM cell culture medium (Figure S6). Additionally, toxicity was evaluated for selected compounds against a series of immortalized cell lines (Figure S7 and Table S2) to inform us about appropriate dosing during the cellular fluorescence experiments. 
A

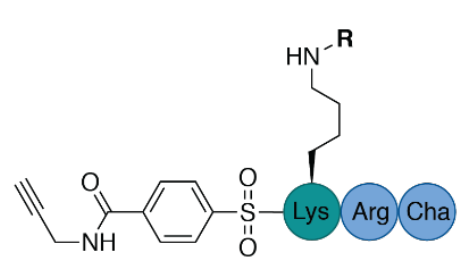

$\mathbf{R}=$ H $_{\mathrm{Me}}^{\mathrm{S}}$

1

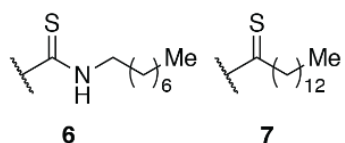

6
$\mathrm{CF}_{3}$

3

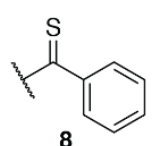

8

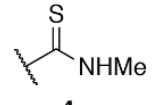

$\underbrace{\mathrm{H}} \mathrm{H}^{\mathrm{S}} \mathrm{H}_{2}^{\mathrm{Me}}$

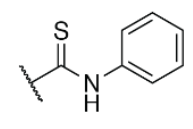

9

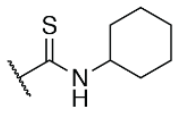

10
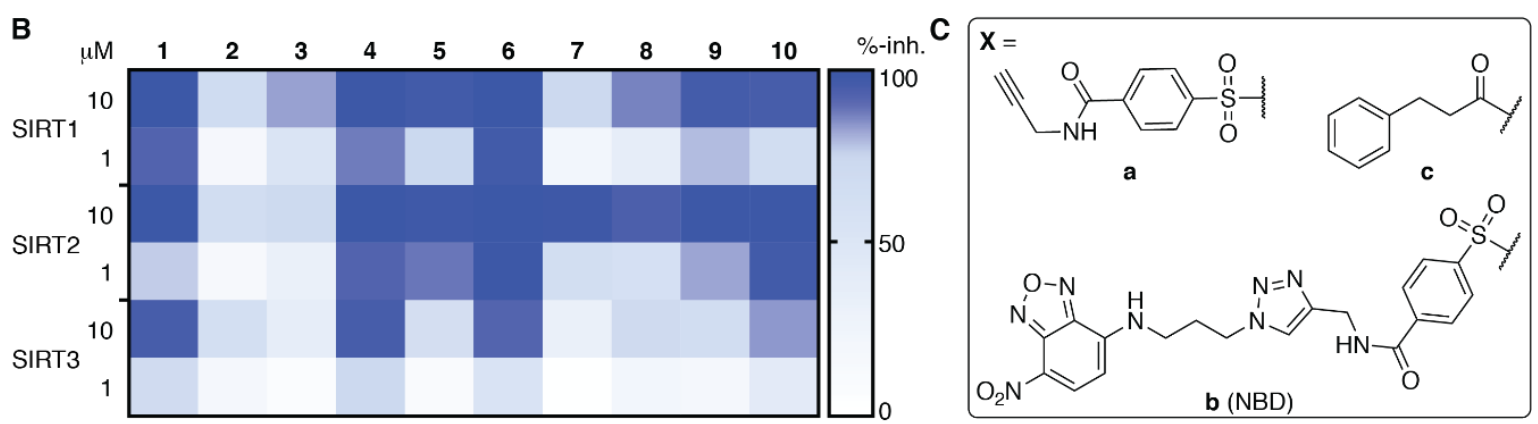

D
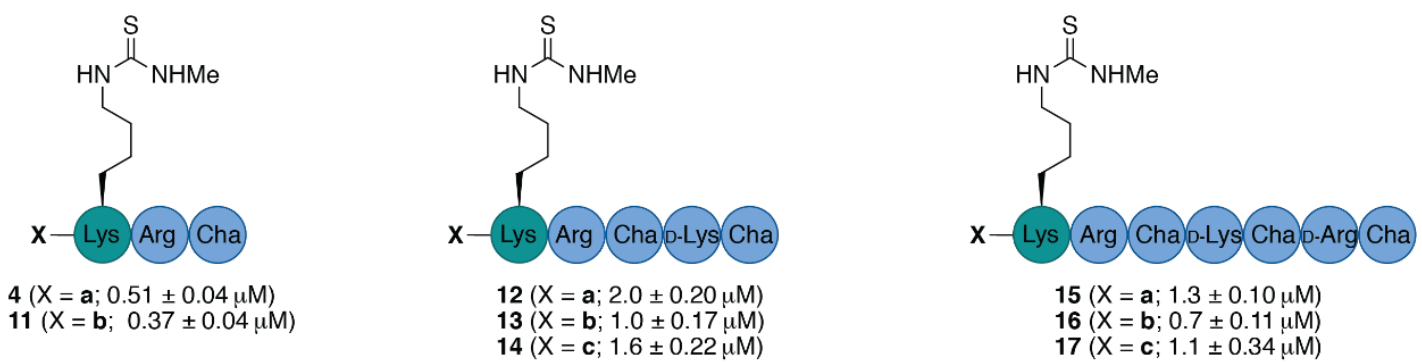

Figure 2. Structure-activity relationship of inhibitors against SIRT1-3. (A) Structures of compounds 1-10. (B) Heatmap summarizing potencies of compounds 1-10 against SIRT1-3 based on \%-inhibition. All assays were performed at least twice in duplicate and the values can be found in Table S1. (C) Structures of Nterminal functional groups introduced in compounds 11-17. (D) Structures and $\mathrm{IC}_{50}$ values and standard deviations recorded for compounds 11-17 based on a minimum of two individual assays performed in duplicate. Further information and selectivity data can be found in Figure S5 and Table S1.

\section{Mitochondrial localization}

The cellular localization of compounds 11, 13, and 16 was first evaluated in HeLa cells (Figure 3 and Figure S8). Not surprisingly, the trimeric scaffold 11 did not display satisfactory cellular or mitochondrial uptake (Figure $3 \mathrm{~A}$ ), which is in line with a previous report where tetramers were the smallest motifs shown to induce targeting of the mitochondria. ${ }^{37}$ Both the pentameric (13) and heptameric (16) probes were taken up by the cells and showed excellent overlap with the MitoTracker ${ }^{\mathrm{TM}}$ co-staining dye (Figure 3B,C). When analyzing the fraction of cells with excellent overlap, it was evident that the heptameric inhibitor (16) was superior to the pentameric inhibitor (13). However, all three NBD-conjugated inhibitors were photo-bleaching within minutes, making the analysis challenging. Therefore, we investigated the more efficient heptameric inhibitor, using a selection of alternative fluorophores, chosen based on their reported bleaching properties, size, charge, and commercial availability. The aim being to identify a fluorophore with better performance, 
which could be readily incorporated without altering the properties of the inhibitor too drastically. Unfortunately, poor cellular uptake was observed for the ATTO-containing analog (S11) (Figure S8). Similarly, the EDANS-conjugated analog (S12) showed low permeability, perhaps due to the negative charge present in this fluorophore (Figure S8). An analog containing the widely used BODIPY fluorophore (S13) exhibited excellent photostability and good mitochondrial targeting as judged by the co-localization with MitoTracker dye (Figure S8). However, significant perturbation of a large fraction of the cells was observed, including vesicle formation, possibly through disruption of the mitochondrial membranes. Finally, 2-aminobenzoyl (Abz), which offers a minimal structural modification of the inhibitor due to its small size, was introduced to give compound S14. Although this fluorophore is less powerful with a quantum yield of 0.6 for the free acid, ${ }^{53}$ compared to 0.94 for BODIPY, ${ }^{54}$ it exhibited cellular uptake and did show overlap with the MitoTracker dye, indicating mitochondrial targeting of compound S14 (Figure S8).

A

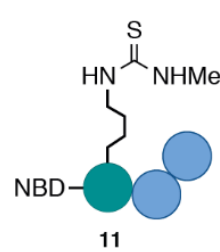

B

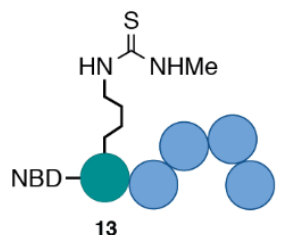

C

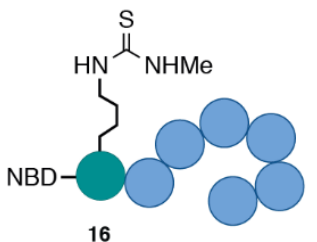

DAP
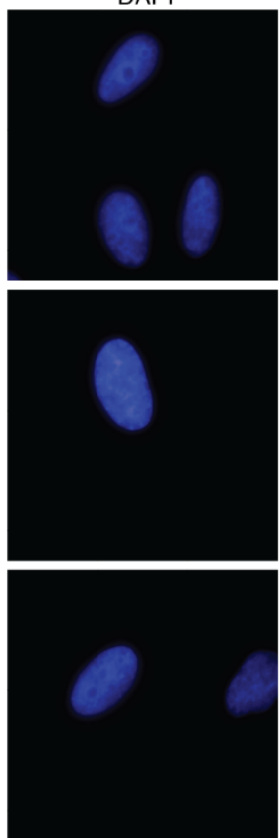

MitoTracker
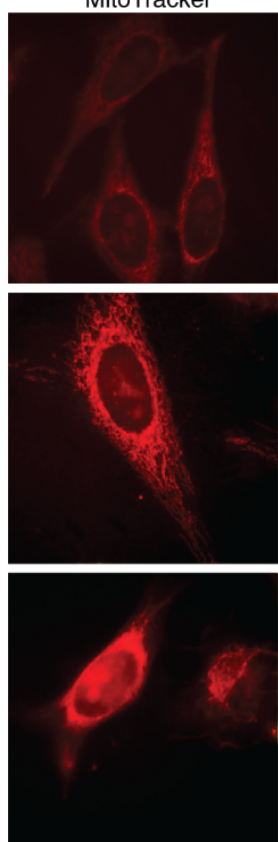

SIRT3 inhibitor

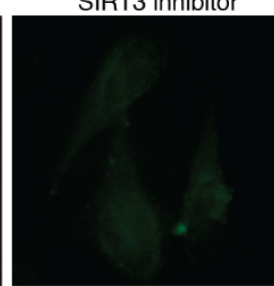

Mitochondrial overlay
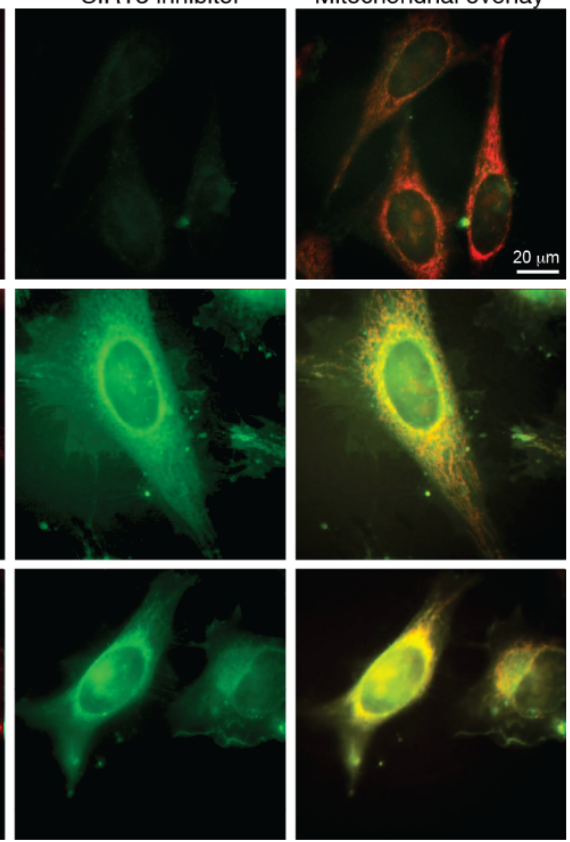

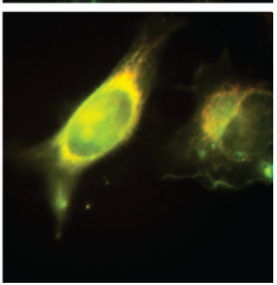

Figure 3. Mitochondrial targeting of fluorophore labeled inhibitors in HeLa cells, determined by co-staining with MitoTrackerTM. (A) NBD-labeled trimer (11); Pearson correlation coefficient $(r)=-0.66$. (B) NBD-labeled pentamer (13); Pearson correlation coefficient $(r)=0.59$. (C) NBD-labeled heptamer (16); Pearson correlation coefficient $(r)=0.46$.

Brightfield images indicated primarily healthy HeLa cells after treatment with most fluorophoreconjugated (Figure S8) as well as the non-fluorophore-conjugated compounds (14 and 17; Figure S9). However, slightly increased toxicity was observed for the heptameric scaffolds and substantial changes to the cell morphology were visible for the BODIPY-conjugated compound as also indicated 
by the fluorescence images discussed above. As expected, the control peptide NBD-TAT (S15) was distributed evenly within the cells with no significant co-localization to the mitochondria and no indication of toxicity in the brightfield images (Figure S8).

Taken together, the data strongly suggest that inhibitors, based on both pentameric and heptameric mitochondria-targeting peptides, are indeed shuttled to the mitochondria in HeLa cells in culture.

\section{Selective targeting of SIRT3 in HEK293T cells in culture}

With the compounds based on the longer heptapeptide mitochondria-targeting sequence showing convincing localization to the mitochondria, we were interested in investigating whether SIRT3 was inhibited selectively in cells. Cellular studies were performed with the non-fluorescent compound 17,

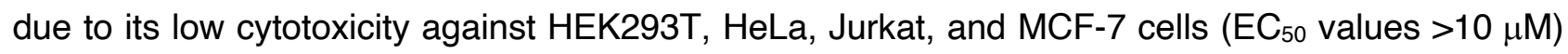
(Figure S7 and Table S2), its low extend of compromising the cell morphology according to brightfield microscopic images of treated HeLa cells (Figure S9), as well as its stability in growth medium (Figure S6). First, we chose the documented mitochondrial protein target MnSOD, for which the degree of acetylation of lysine 68 (K68) has been shown to be regulated by SIRT3.26, 27, 55, 56 Inspired by the work of Meier and co-workers on non-enzymatic acylation, ${ }^{57}$ we developed a novel mitochondrial targeting acetylating agent (18; Scheme 1) to be used as a positive control (see Scheme S4 for synthesis and structure). Gratifyingly, compound 18 showed a significant increase in MnSOD (K68) acetylation in mitochondria enriched fractions of HEK293T lysates. Similarly, cells treated with compound 17 showed a significant increase in MnSOD (K68) acetylation, using $10 \mu \mathrm{M}$ of the inhibitor (Figure 4A, Figure S10, and Figure S11).

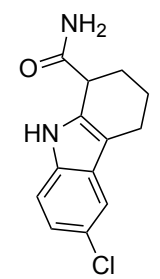

EX-527
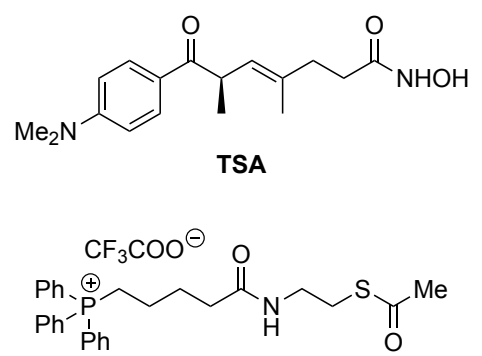

18

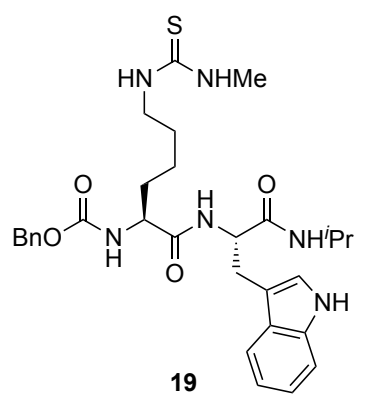

19

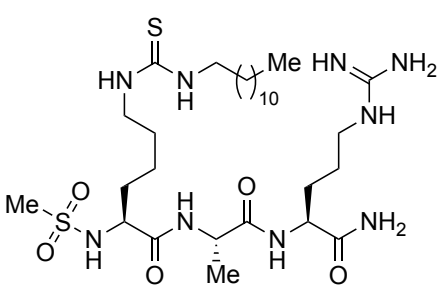

20

Scheme 1. Structures of EX527, TSA, and compounds 18-20.33, 35, 49

Finally, we addressed whether the targeting of SIRT3 was also selective over SIRT1 and 2 in HEK293T cells. For SIRT1, we chose the well documented target $\mathrm{p} 53^{51,58,59}$ and analyzed the levels of acetylated p53 (K382) in HEK293T cells treated with compound 17, compared to the known SIRT1 inhibitors EX-527 $\mathbf{6}^{60}$ and $\mathbf{1 9}{ }^{33,49}$ as positive controls (Scheme 1, Figure 4B, and Figure S12). Although, 
compounds 17, EX-527, and 19 are equipotent against SIRT1 in vitro, ${ }^{49}$ the effect on p53 acetylation (K382) by compound $\mathbf{1 7}$ is significantly lower than the positive control compounds (Figure 4B), suggesting a high degree of selectivity for SIRT3 over SIRT1 in cells.

A
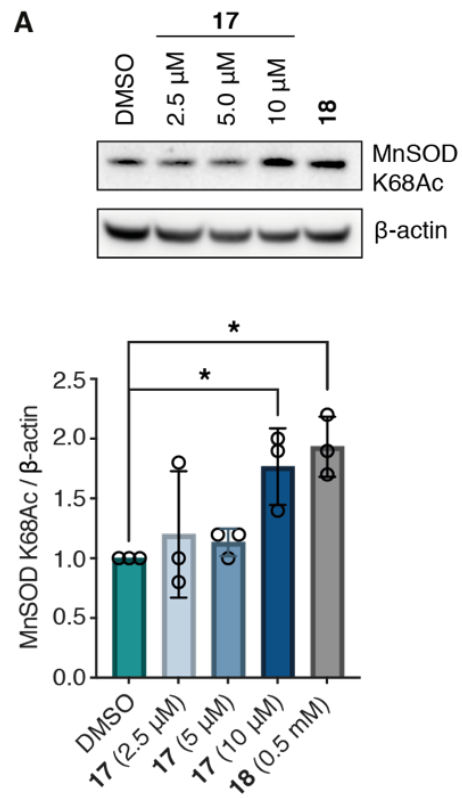

C

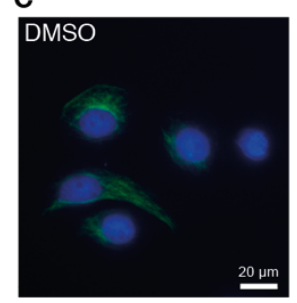

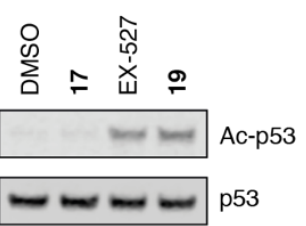
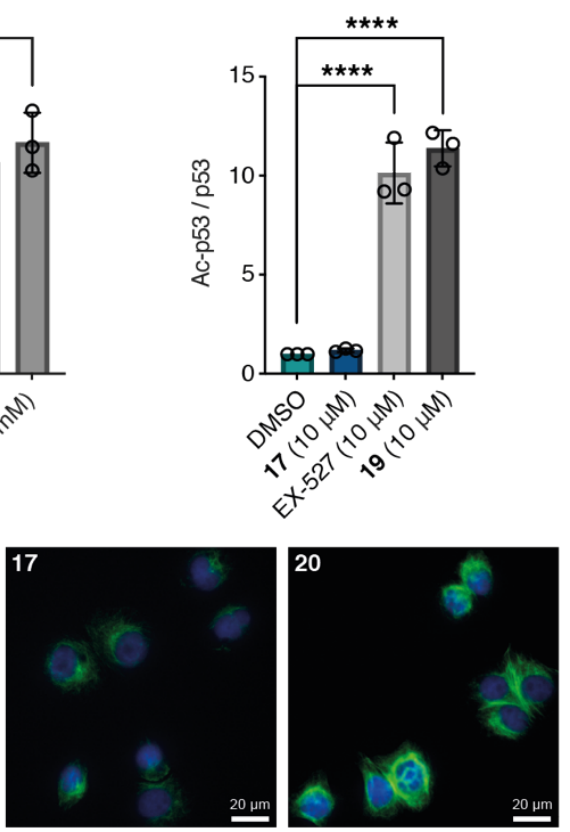

Figure 4. Inhibition of SIRT3 in HEK293T cells. (A) Representative western blot and quantification data showing dose-dependent effect of $\mathbf{1 7}$ on acetylation of the documented mitochondrial SIRT3 target MnSOD compared to the positive control $18(0.5 \mathrm{mM})(n=3$; for full blots and replicates, see Figure $S 10$ and $S 11)$. (B) Evaluation of off-target effect of $17(10 \mu \mathrm{M})$ on p53 acetylation compared to positive controls EX-527 $(10 \mu \mathrm{M})$ and $19(10 \mu \mathrm{M})$ by western blot. Cells were cotreated with TSA $(1 \mu \mathrm{M})$ to abolish interfering activity of $\mathrm{Zn}^{2+-}$ dependent HDACs ( $n=3$; for conditions, full blots, and replicates, see Figure S12). (C) Immunofluorescence investigation of the effect of $\mathbf{1 7}(10 \mu \mathrm{M})$ on $\alpha$-tubulin acetylation compared to DMSO and $20(10 \mu \mathrm{M})$ as the positive control. The data are representative images from two individual experiments. For conditions and additional images, see Figure S13. Significance of the levels of Ac-p53 and MnSOD K68Ac were calculated using one-way ANOVA test. Adjusted $p$ values: ns $p>0.05,{ }^{*} p<0.05,{ }^{* *} p<0.01,{ }^{* * *} p<0.001,{ }^{* * * *} p<0.0001$ compared to DMSO treated control.

For SIRT2, we and others have recently reported on the challenges of determining cellular effects on lysine acetylation by performing western blots on whole cell extracts. ${ }^{61}$ We therefore investigated the effect of 17 , on $\alpha$-tubulin acetylation (K40) qualitatively by performing immunofluorescence experiments, comparing to DMSO and $\mathbf{2 0}$ (Scheme 1) as negative and positive controls, respectively 
(Figure 4C and Figure S13). While treatment with compound 20 produced a significant increase in $\alpha$-tubulin acetylation compared to the DMSO control, no change was observed when treating cells with either compound 17, indicating high selectivity towards SIRT3 over SIRT2 in cells as well.

\section{Target engagement in HEK293T cells by cellular thermal shift assay}

To further evaluate whether compound $\mathbf{1 7}$ is directly targeting SIRT3 in living cells, we performed cellular thermal shift assays in HEK293T cells in culture, using immunoblotting for the subsequent analysis (Figure 5 and Figures S14-18). ${ }^{62-64}$. A homologue of compound 17, containing a free lysine residue (17-K), which does not inhibit sirtuin activity was synthesized and tested as a negative control (for synthesis and profiling of 17-K, please consult Scheme S3, Figure S5 and Table S1). Upon treatment with the compounds at various temperatures, the cellular samples were lysed to provide whole cell lysates, which were subjected to SDS gel electrophoresis and western blotting. Analysis of the normalized data led to a statistically significant $(P<0.01)$ shift in thermal stability of SIRT3 for 17 and not 17-K compared to the DMSO control, strongly suggesting specific target engagement of compound 17 with SIRT3 in HEK293T cells in culture. The SIRT2 enzyme did not appear to be stabilized by neither 17 nor 17-K, while positive control compound SirReal2 exhibited stabilization of this enzyme (Figure S15). These findings were in agreement with the evaluation of ability to affect $\alpha$-tubulin acetylation in the immunohistochemical assays discussed above. For SIRT1, on the other hand, compound $\mathbf{1 7}$ appeared to stabilize the enzyme up to a similar temperature as the positive control SIRT1 inhibitor 19 (Figure 5, S14 and S18). This was surprising because we did not record an effect of 17 comparable to 19 in the p53 acetylation assay, which indirectly reports on SIRT1 inhibition in the cells. It should therefore be noted that the thermal shift assay does not report on compound potency or ability to inhibit the enzyme in question, but simply whether the inhibitor binds to the enzyme in a fashion that causes an increase in thermal stability. All the data taken together, therefore shows that both SIRT1 and SIRT3 bind compound 17 in cultured HEK293T cells but only SIRT3 is inhibited by 17 to an extent where downstream effect on a known deacetylation targets is recorded.

\section{Conclusion}

It has been a major challenge to develop inhibitors that selectively target SIRT3 of the class I sirtuin enzymes. Due to the high structural similarity of the active sites of SIRT1-3, small molecule chemotypes have largely failed in producing selective inhibition of SIRT3 over SIRT1 and 2. The differences in the structures of the extended substrate-binding pockets among these three enzymes 
have enabled the development of selective inhibitors of SIRT2 but have not been successfully harnessed to target SIRT1 or SIRT3.

A
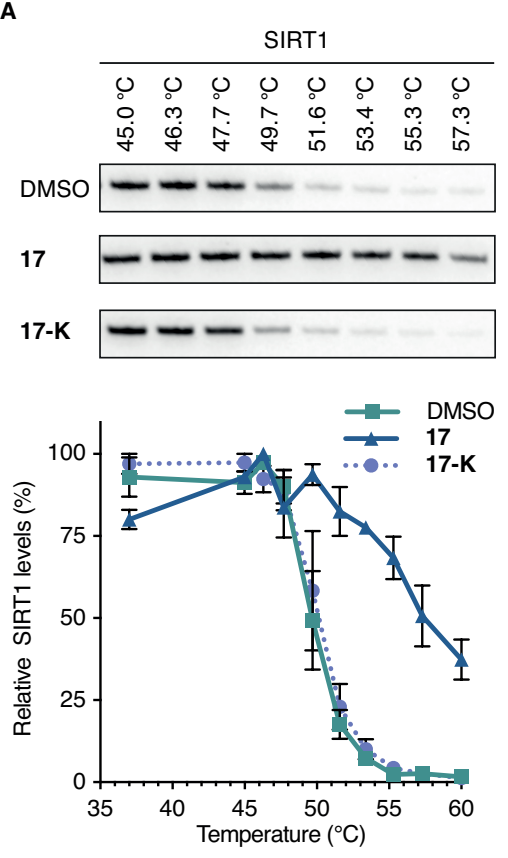

\begin{tabular}{ll} 
SIRT1 & $\mathbf{T}_{\text {agg }}\left({ }^{\circ} \mathbf{C}\right)$ \\
\hline DMSO & $49.8 \pm 0.2$ \\
\hline $\mathbf{1 7}$ & $56.1 \pm 1.2^{\star}$ \\
\hline $\mathbf{1 7 - K}$ & $50.1 \pm 0.3$ \\
\hline
\end{tabular}

B
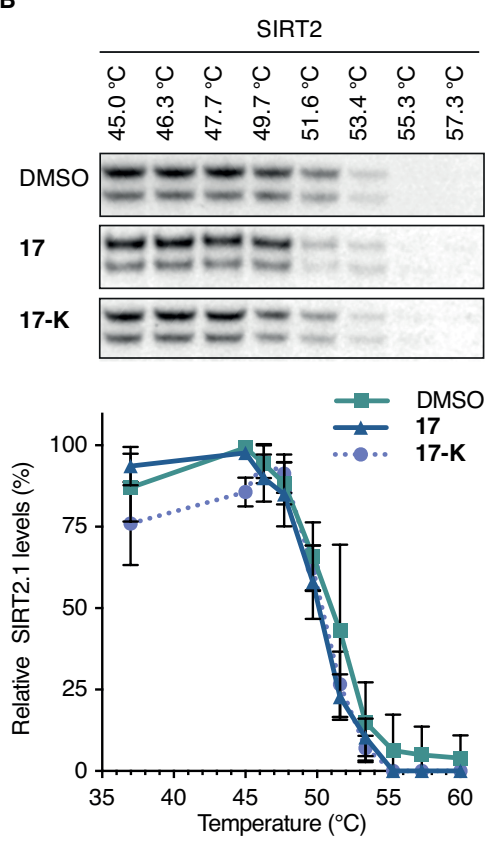

\begin{tabular}{ll} 
SIRT2.1 & $\mathbf{T}_{\text {agg }}\left({ }^{\circ} \mathbf{C}\right)$ \\
\hline DMSO & $51.0 \pm 0.4$ \\
\hline $\mathbf{1 7}$ & $50.2 \pm 0.3$ \\
\hline $\mathbf{1 7 - K}$ & $50.7 \pm 0.3$ \\
\hline
\end{tabular}
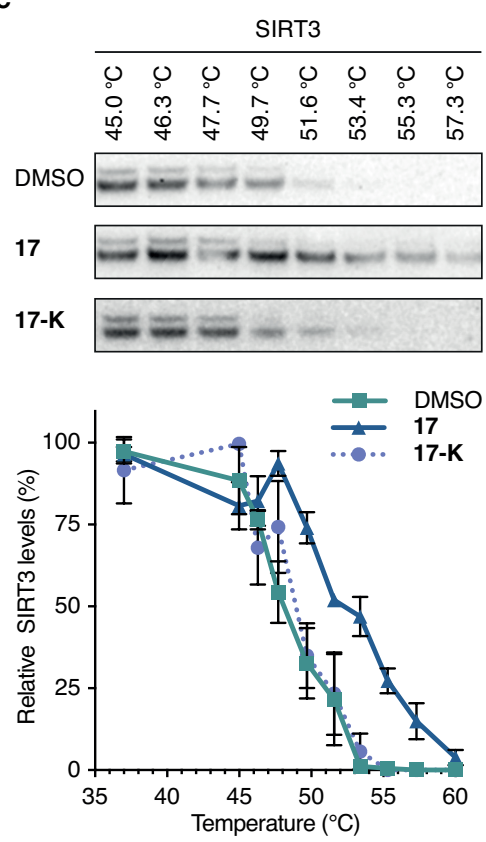

\begin{tabular}{ll} 
SIRT3 & $\mathrm{T}_{\text {agg }}\left({ }^{\circ} \mathbf{C}\right)$ \\
\hline DMSO & $48.6 \pm 0.3$ \\
\hline 17 & $53.1 \pm 0.8^{\star \star}$ \\
\hline $17-\mathrm{K}$ & $49.3 \pm 0.4$ \\
\hline
\end{tabular}

Figure 5. Representative blots, plots, and $T_{\text {agg }}$ values from cellular thermal shift assay evaluation of 17 vs. 17K, with DMSO control. (A) Investigation of SIRT1 levels from HEK293T cells upon treatment with DMSO, 17 $(10 \mu \mathrm{M})$ or $17-\mathrm{K}(10 \mu \mathrm{M})$ and heating to the specified temperatures. $(\mathrm{n}=3$; for full dataset see Figure $\mathrm{S} 14$ and for full blots and replicates, see full western blot section. (B) Investigation of SIRT2 levels from HEK293T cells upon treatment with DMSO, $17(10 \mu \mathrm{M})$ or $\mathbf{1 7 - K}(10 \mu \mathrm{M})$ and heating to the specified temperatures. $(\mathrm{n}=3$; for full dataset see Figure S15 and Supporting Information for full blots and replicates). (C) Investigation of SIRT3 levels from HEK293T cells upon treatment with DMSO, $17(10 \mu \mathrm{M})$ or 17-K $(10 \mu \mathrm{M})$ and heating to the specified temperatures ( $n=5$ for DMSO and compound 17 treatments. $n=3$ for compound 17-K treatments; for full dataset see Figures S16, S17 and Supporting Information for full blots and replicates). Significance of the CETSA shifts were calculated using unpaired t-test of $\mathrm{T}_{\text {agg }}$ values from independent experiments. Adjusted $p$ values: ${ }^{*} p<0.05,{ }^{* *} p<0.01$ compared to DMSO treated control.

Here, we developed compounds based on a different strategy that takes advantage of the differential sub-cellular localization of the three class I sirtuins. In work reported during the finalization of our study, a commonly used mitochondrial targeting motif (the triphenylphosphonium group) was attached to a SIRT2 inhibitor, to inhibit SIRT3 in the mitochondria rather than SIRT1 and SIRT2 in the nucleus and cytosol, respectively. ${ }^{65}$ The chemotypes developed in the present study, however, have a fundamentally different architecture and includes optimization of their selectivity profiles to 
dial down affinity for SIRT1 and SIRT2. Our design was predicated on the fusion of attributes from mechanism-based class I sirtuin inhibitors with mitochondria-targeting peptides. After succeeding in the achievement of potent enzyme inhibition in vitro, we optimized the mitochondria-targeting properties and secured compound stability together with limited toxicity. The resulting probe compound exhibited direct engagement of SIRT3 in the mitochondria of cells in culture by an inhibitor molecule for the first time and the downstream acetylation level of the documented SIRT3 target MnSOD was increased.

We expect that this novel probe will enable investigation of the function of SIRT3 with unprecedented precision and thus help uncover the potential for development of future therapeutics targeting this enzyme. Finally, our results provide a framework that may be exploited for the targeting of other mitochondrial proteins, including the mitochondrial sirtuin isoforms, SIRT4 and SIRT5, by incorporating alternative acyl group mimics that are selectively targeted by these enzymes.

\section{ORCID}

Kathrin Sten Troelsen: 0000-0002-8734-533X

Michael Bæk:0000-0001-7416-9421

Alexander L. Nielsen: 0000-0003-1195-0143

Andreas S. Madsen: 0000-0001-7283-2090

Nima Rajabi: 0000-0002-9509-7540

Christian Adam Olsen: 0000-0002-2953-8942

\section{Conflict of interest}

The authors declare no conflict of interest.

\section{Acknowledgements}

We thank Julie E. Bolding for assistance with mitochondrial enrichment and donation of recombinant SIRT7. This work was supported by the Lundbeck foundation (PhD Fellowship grant R218-20161277 and Running Cost grant R289-2018-2074), the Danish Independent Research Council-Natural Sciences (Grant No. 6108-00166B), the Carlsberg Foundation (2013-01-0333, CF15-011, and CF18-0442), the Novo Nordisk Foundation (NNF170C0029464), and the European Research Council (ERC-CoG-725172-SIRFUNCT).

\section{References}

1. M. C. Haigis and D. A. Sinclair, Annu. Rev. Pathol., 2010, 5, 253-295. 
2. A. Chalkiadaki and L. Guarente, Nat. Rev. Cancer, 2015, 15, 608-624.

3. N. Rajabi, I. Galleano, A. S. Madsen and C. A. Olsen, in Sirtuins in Health and Disease, ed. W. Zheng, Academic Press, 2018, vol. 154, pp. 25-69.

4. H. Jiang, S. Khan, Y. Wang, G. Charron, B. He, C. Sebastian, J. Du, R. Kim, E. Ge, R. Mostoslavsky, H. C. Hang, Q. Hao and H. Lin, Nature, 2013, 496, 110.

5. J. L. Feldman, J. Baeza and J. M. Denu, J. Biol. Chem., 2013, 288, 31350-31356.

6. A. S. Madsen, C. Andersen, M. Daoud, K. A. Anderson, J. S. Laursen, S. Chakladar, F. K. Huynh, A. R. Colaco, D. S. Backos, P. Fristrup, M. D. Hirschey and C. A. Olsen, J. Biol. Chem., 2016, 291, 7128-7141.

7. D. Rauh, F. Fischer, M. Gertz, M. Lakshminarasimhan, T. Bergbrede, F. Aladini, C. Kambach, C. F. Becker, J. Zerweck, M. Schutkowski and C. Steegborn, Nat. Commun., 2013, 4, 2327.

8. L. L. Yang, H. L. Wang, Y. H. Yan, S. Liu, Z. J. Yu, M. Y. Huang, Y. Luo, X. Zheng, Y. Yu and G. B. Li, Eur J Med Chem, 2020, 192, 112201.

9. R. H. Houtkooper, E. Pirinen and J. Auwerx, Nat. Rev. Mol. Cell Biol., 2012, 13, 225-238.

10. M. Schiedel, D. Robaa, T. Rumpf, W. Sippl and M. Jung, Med. Res. Rev., 2018, 38, 147-200.

11. M. S. Bonkowski and D. A. Sinclair, Nat. Rev. Mol. Cell Biol., 2016, 17, 679-690.

12. Y. Jiang, J. Liu, D. Chen, L. Yan and W. Zheng, Trends Pharmacol. Sci., 2017, 38, 459-472.

13. J. Du, Y. Zhou, X. Su, J. J. Yu, S. Khan, H. Jiang, J. Kim, J. Woo, J. H. Kim, B. H. Choi, B. He, W. Chen, S. Zhang, R. A. Cerione, J. Auwerx, Q. Hao and H. Lin, Science, 2011, 334, 806.

14. A. S. Madsen and C. A. Olsen, J. Med. Chem., 2012, 55, 5582-5590.

15. K. A. Anderson, F. K. Huynh, K. Fisher-Wellman, J. D. Stuart, B. S. Peterson, J. D. Douros, G. R. Wagner, J. W. Thompson, A. S. Madsen, M. F. Green, R. M. Sivley, O. R. Ilkayeva, R. D. Stevens, D. S. Backos, J. A. Capra, C. A. Olsen, J. E. Campbell, D. M. Muoio, P. A. Grimsrud and M. D. Hirschey, Cell Metab., 2017, 25, 838-855 e815.

16. M. Pannek, Z. Simic, M. Fuszard, M. Meleshin, D. Rotili, A. Mai, M. Schutkowski and C. Steegborn, Nat. Commun., 2017, 8, 1513.

17. M. Tan, C. Peng, K. A. Anderson, P. Chhoy, Z. Xie, L. Dai, J. Park, Y. Chen, H. Huang, Y. Zhang, J. Ro, G. R. Wagner, M. F. Green, A. S. Madsen, J. Schmiesing, B. S. Peterson, G. Xu, O. R. Ilkayeva, M. J. Muehlbauer, T. Braulke, C. Muhlhausen, D. S. Backos, C. A. Olsen, P. J. McGuire, S. D. Pletcher, D. B. Lombard, M. D. Hirschey and Y. Zhao, Cell Metab., 2014, 19, 605-617.

18. C. Roessler, C. Tuting, M. Meleshin, C. Steegborn and M. Schutkowski, J. Med. Chem., 2015, 58, 7217-7223.

19. C. Roessler, T. Nowak, M. Pannek, M. Gertz, G. T. Nguyen, M. Scharfe, I. Born, W. Sippl, C. Steegborn and M. Schutkowski, Angew Chem Int Ed, 2014, 53, 10728-10732.

20. B. H. Ahn, H. S. Kim, S. Song, I. H. Lee, J. Liu, A. Vassilopoulos, C. X. Deng and T. Finkel, Proc. Natl. Acad. Sci. USA, 2008, 105, 14447-14452.

21. C. Schlicker, M. Gertz, P. Papatheodorou, B. Kachholz, C. F. Becker and C. Steegborn, J. Mol. Biol., 2008, 382, 790-801.

22. H. Cimen, M. J. Han, Y. Yang, Q. Tong, H. Koc and E. C. Koc, Biochemistry, 2010, 49, 304311.

23. M. D. Hirschey, T. Shimazu, E. Goetzman, E. Jing, B. Schwer, D. B. Lombard, C. A. Grueter, C. Harris, S. Biddinger, O. R. Ilkayeva, R. D. Stevens, Y. Li, A. K. Saha, N. B. Ruderman, J. R. Bain, C. B. Newgard, R. V. Farese, Jr., F. W. Alt, C. R. Kahn and E. Verdin, Nature, 2010, 464, 121-125.

24. T. Shimazu, M. D. Hirschey, L. Hua, K. E. Dittenhafer-Reed, B. Schwer, D. B. Lombard, Y. Li, J. Bunkenborg, F. W. Alt, J. M. Denu, M. P. Jacobson and E. Verdin, Cell Metab., 2010, 12, 654-661.

25. A. S. Bause and M. C. Haigis, Exp. Gerontol., 2013, 48, 634-639. 
26. X. Qiu, K. Brown, M. D. Hirschey, E. Verdin and D. Chen, Cell Metab., 2010, 12, 662-667.

27. R. Tao, M. C. Coleman, J. D. Pennington, O. Ozden, S. H. Park, H. Jiang, H. S. Kim, C. R. Flynn, S. Hill, W. Hayes McDonald, A. K. Olivier, D. R. Spitz and D. Gius, Mol. Cell, 2010, 40, 893-904.

28. B. M. Hirsch, C. A. Gallo, Z. Du, Z. Wang and W. Zheng, MedChemComm, 2010, 1, 233238.

29. B. C. Smith and J. M. Denu, Biochemistry, 2007, 46, 14478-14486.

30. B. C. Smith and J. M. Denu, J. Biol. Chem., 2007, 282, 37256-37265.

31. B. C. Dancy, S. A. Ming, R. Papazyan, C. A. Jelinek, A. Majumdar, Y. Sun, B. M. Dancy, W. J. Drury, 3rd, R. J. Cotter, S. D. Taverna and P. A. Cole, J. Am. Chem. Soc., 2012, 134, 5138-5148.

32. N. Rajabi, M. Auth, K. R. Troelsen, M. Pannek, D. P. Bhatt, M. Fontenas, M. D. Hirschey, C. Steegborn, A. S. Madsen and C. A. Olsen, Angew Chem Int Ed, 2017, 56, 14836-14841.

33. S. V. Olesen, N. Rajabi, B. Svensson, C. A. Olsen and A. S. Madsen, Biochemistry, 2018, 57, 3903-3915.

34. M. Bæk, P. Martin-Gago, J. S. Laursen, J. L. H. Madsen, S. Chakladar and C. A. Olsen, Chem Eur J, 2020, 26, 3862-3869.

35. A. L. Nielsen, N. Rajabi, N. Kudo, K. Lundø, M. Fontenas, A. Lucidi, A. S. Madsen, M. Yoshida and C. A. Olsen, bioRxiv, 2020, DOI: 10.1101/2020.1103.1120.000380.

36. M. C. Frantz and P. Wipf, Environ. Mol. Mutagen., 2010, 51, 462-475.

37. K. L. Horton, K. M. Stewart, S. B. Fonseca, Q. Guo and S. O. Kelley, Chem Biol, 2008, 15, 375-382.

38. L. F. Yousif, K. M. Stewart and S. O. Kelley, ChemBioChem, 2009, 10, 1939-1950.

39. K. L. Horton, M. P. Pereira, K. M. Stewart, S. B. Fonseca and S. O. Kelley, ChemBioChem, 2012, 13, 476-485.

40. D. G. Fatkins, A. D. Monnot and W. Zheng, Bioorg. Med. Chem. Lett., 2006, 16, 3651-3656.

41. B. Chen, W. Zang, J. Wang, Y. Huang, Y. He, L. Yan, J. Liu and W. Zheng, Chem. Soc. Rev., 2015, 44, 5246-5264.

42. D. Wegener, F. Wirsching, D. Riester and A. Schwienhorst, Chem Biol, 2003, 10, 61-68.

43. T. Kosciuk, I. R. Price, X. Zhang, C. Zhu, K. N. Johnson, S. Zhang, S. L. Halaby, G. P. Komaniecki, M. Yang, C. J. DeHart, P. M. Thomas, N. L. Kelleher, J. C. Fromme and H. Lin, Nat. Commun., 2020, 11, 1067.

44. A. S. Farooqi, J. Y. Hong, J. Cao, X. Lu, I. R. Price, Q. Zhao, T. Kosciuk, M. Yang, J. J. Bai and H. Lin, J. Med. Chem., 2019, 62, 4131-4141.

45. C. W. Tornoe, C. Christensen and M. Meldal, J. Org. Chem., 2002, 67, 3057-3064.

46. V. V. Rostovtsev, L. G. Green, V. V. Fokin and K. B. Sharpless, Angew Chem Int Ed, 2002, 41, 2596-2599.

47. J. L. Feldman, K. E. Dittenhafer-Reed, N. Kudo, J. N. Thelen, A. Ito, M. Yoshida and J. M. Denu, Biochemistry, 2015, 54, 3037-3050.

48. X. Bao, Y. Wang, X. Li, X. M. Li, Z. Liu, T. Yang, C. F. Wong, J. Zhang, Q. Hao and X. D. Li, Elife, 2014, 3, e02999.

49. N. Rajabi, A. L. Nielsen and C. A. Olsen, ACS Med. Chem. Lett., 2020, 11, 1886-1892.

50. B. Chen, J. Wang, Y. Huang and W. Zheng, Bioorg Med Chem Lett, 2015, 25, 3481-3487.

51. H. Jing, J. Hu, B. He, Y. L. Negron Abril, J. Stupinski, K. Weiser, M. Carbonaro, Y. L. Chiang, T. Southard, P. Giannakakou, R. S. Weiss and H. Lin, Cancer Cell, 2016, 29, 297-310.

52. M. Zessin, Z. Kutil, M. Meleshin, Z. Nováková, E. Ghazy, D. Kalbas, M. Marek, C. Romier, W. Sippl, C. Bařinka and M. Schutkowski, Biochemistry, 2019, 58, 4777-4789.

53. A. S. Culf, H. Yin, S. Monro, A. Ghosh, D. A. Barnett, R. J. Ouellette, M. Cuperlovic-Culf and S. A. McFarland, Bioorg Med Chem, 2016, 24, 929-937.

54. L. D. Lavis and R. T. Raines, ACS Chem. Biol., 2008, 3, 142-155. 
55. Y. Chen, J. Zhang, Y. Lin, Q. Lei, K. L. Guan, S. Zhao and Y. Xiong, EMBO Rep., 2011, 12, 534-541.

56. C. He, J. M. Danes, P. C. Hart, Y. Zhu, Y. Huang, A. L. de Abreu, J. O'Brien, A. J. Mathison, B. Tang, J. M. Frasor, L. M. Wakefield, D. Ganini, E. Stauder, J. Zielonka, B. N. Gantner, R. A. Urrutia, D. Gius and M. G. Bonini, Proc. Natl. Acad. Sci. USA, 2019, 116, 23534-23541.

57. R. A. Kulkarni, A. J. Worth, T. T. Zengeya, J. H. Shrimp, J. M. Garlick, A. M. Roberts, D. C. Montgomery, C. Sourbier, B. K. Gibbs, C. Mesaros, Y. C. Tsai, S. Das, K. C. Chan, M. Zhou, T. Andresson, A. M. Weissman, W. M. Linehan, I. A. Blair, N. W. Snyder and J. L. Meier, Cell Chem. Biol., 2017, 24, 231-242.

58. T. Suzuki, T. Asaba, E. Imai, H. Tsumoto, H. Nakagawa and N. Miyata, Bioorg Med Chem Lett, 2009, 19, 5670-5672.

59. H. Vaziri, S. K. Dessain, E. N. Eaton, S.-I. Imai, R. A. Frye, T. K. Pandita, L. Guarente and R. A. Weinberg, Cell, 2001, 107, 149-159.

60. A. D. Napper, J. Hixon, T. McDonagh, K. Keavey, J. F. Pons, J. Barker, W. T. Yau, P. Amouzegh, A. Flegg, E. Hamelin, R. J. Thomas, M. Kates, S. Jones, M. A. Navia, J. O. Saunders, P. S. DiStefano and R. Curtis, J. Med. Chem., 2005, 48, 8045-8054.

61. T. Rumpf, M. Schiedel, B. Karaman, C. Roessler, B. J. North, A. Lehotzky, J. Olah, K. I. Ladwein, K. Schmidtkunz, M. Gajer, M. Pannek, C. Steegborn, D. A. Sinclair, S. Gerhardt, J. Ovadi, M. Schutkowski, W. Sippl, O. Einsle and M. Jung, Nat. Commun., 2015, 6, 6263.

62. D. Martinez Molina, R. Jafari, M. Ignatushchenko, T. Seki, E. A. Larsson, C. Dan, L. Sreekumar, Y. Cao and P. Nordlund, Science, 2013, 341, 84-87.

63. D. Martinez Molina and P. Nordlund, Annu Rev Pharmacol Toxicol, 2016, 56, 141-161.

64. R. Jafari, H. Almqvist, H. Axelsson, M. Ignatushchenko, T. Lundback, P. Nordlund and D. Martinez Molina, Nat. Protoc., 2014, 9, 2100-2122.

65. M. Li, Y. L. Chiang, C. A. Lyssiotis, M. R. Teater, J. Y. Hong, H. Shen, L. Wang, J. Hu, H. Jing, Z. Chen, N. Jain, C. Duy, S. J. Mistry, L. Cerchietti, J. R. Cross, L. C. Cantley, M. R. Green, H. Lin and A. M. Melnick, Cancer Cell, 2019, 35, 916-931 e919.

\section{ToC Graphic}

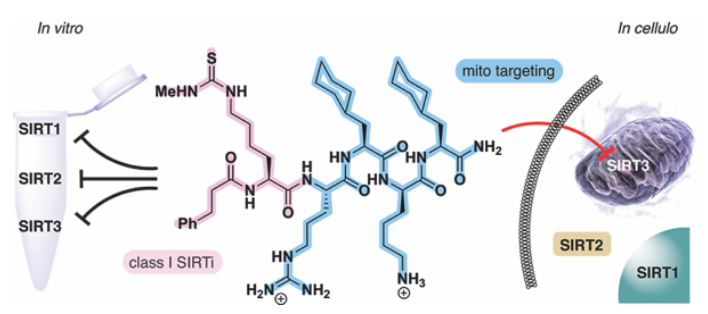

